\title{
The scientific case for muon physics at a neutrino factory
}

\section{SACHA DAVIDSON*广}

IPNL

E-mail: s.davidson@ipnl.in2p3.fr

This proceedings asks simple questions about what New Physics can be learned from muon precision experiments, in the era of the LHC. The New Physics is parametrised by an effective Lagrangian, and the focus is on lepton flavour violating processes, with small mention of $(g-2)_{\mu}$.

10th International Workshop on Neutrino Factories, Super beams and Beta beams

June 30 - July 52008

Valencia, Spain

*Speaker.

$\dagger$ The talk this manuscript is based on, was originally attributed to A Czarnecki, who could not attend the conference. I am grateful to AC for assistance in writing the talk, on a subject he knows far better than I. I also thank the organisers, on one hand for a most excellently organised and interesting conference, and also, perversely, for the "opportunity" to learn a great deal very quickly about something new. 


\section{Introduction}

The muon has a long history in precise determination of model parameters, and in searching for New Physics (NP). It continues in both roles: NP is hinted at in $(g-2)_{\mu}$ and hoped for in $\mu \rightarrow e \gamma$, and, should signatures of NP be found at the LHC, muon physics could (hopefully) contribute to distinguishing amoung the possible models.

The science case for muon physics at a $v$ Factory was presented in 2001 by the CERN working group study [2] (a more recent discussion can be found in [3]). A useful review of results and possibilities of all aspects of precision muon physics can be found on [1]. A recent compendium of bounds on dipole and four-fermion operators involving leptons, can be found in [4]. The constraints on New Physics from the muon - photon dipole interactions (see eqn 4.1; these loops can be sensitive to Beyond-the-Standard-Model physics) can be found in Hisano's contributions to $v$ Factory proceedings of last year [11]. Restrictive experimental constraints come from $(g-2)_{\mu}$, the electric dipole moment of the muon, and $\mu \rightarrow e \gamma$. The discrepancy between theory and experiment in the muon magnetic moment $(g-2)_{\mu}$ is discussed in the contribution of L Roberts to these proceedings. First results from the $\mu \rightarrow e \gamma$ experiment MEG are expected soon; see the WG4 contribution of D Nicolo. For lack of space, I do not discuss electric dipole moments (K Kirch in WG4), muonium, or Fermi decay (P Kammel, A Grossheim in WG4).

Perhaps the most significant change, since $[1,2]$, is the date: a source producing $10^{18}$ muons/yr is still in the future, but the LHC is turning on now. To write this talk, I asked myself questions (which reappear as section headers); the most basic of which was "what is the role of muon physics, in the era of the LHC?"

\section{Why is muon physics interesting?}

The existence of "new" physics (NP) Beyond the Standard Model (BSM) is required by various observations, such as Dark Matter, the Baryon Asymmetry of the Universe, and, of particular interest here, neutrino masses. These masses demonstrate that there is NP in the lepton sector, which at some level should appear in precision muon observations. There are also theoretical arguments, such as the "hierarchy problem", which suggest NP close to the electroweak scale.

One hopes that upcoming data, in astrophysics, low energy precision experiments, and colliders, will contain footprints of NP. Precision muon physics can play two roles in the interpretation of this data. First, it can be sensitive to to higher mass scales and smaller couplings than the LHC (the well-known "complementarity" between collider and precision searches). Secondly, when the sensitivity of muon experiments overlaps with some other data (such as from the LHC), muon results may play an important role in distinguishing models and determining their parameters. (Similiar to the electroweak precision fit, where of the three inputs, $G_{F}, \alpha_{e m}$, were from low energy.)

\section{The effective Lagrangian $\mathscr{L}_{\text {eff }}$}

At energies well below the new physics scale $\Lambda$, the effects of the NP can be described by an effective Lagrangian. It is a sum of non-renormalisable operators, constructed out of Standard 
Model fields, and respecting SM gauge symmetries:

$$
\Delta \mathscr{L}_{e f f}=\sum_{d \geq 5} \sum_{n} \frac{C^{(n)}}{\Lambda^{d-4}} O_{n}\left(H,\{\psi\}, A_{\mu}, \ldots\right)+\text { h.c. }
$$

These new operators can be classified by their mass dimension $4+n$, where $n$ is the number of powers of $\Lambda$ in the denominator: at $1 / \Lambda$ there is only the neutrino mass operator, at $\mathscr{O}\left(1 / \Lambda^{2}\right)$ are various operators relevant to precision muon experiments such as the dipole operator (eqn (4.1)), and four fermion operators (eqn (6.1)). Non-Standard Interactions, of particular interest to future neutrino beams, usually appear at dimension 8 .

The coefficients $\frac{C^{(n)}}{\Lambda^{d-4}}$ are closely related to the effective coupling constant of the Feynman diagram associated to the operator. They can have contributions from the SM (for instance $G_{F}$ ) and from NP we hope to measure. The dimensionless constant $C$ in eqn (3.1) is a product of coupling constants, probably $<1$.

\section{Dimension six operators with a muon}

The relevant operators for precision muon physics are certain four fermion operators, mentioned at eqn (6.1), and the dipoles:

$$
\left\{\frac{C^{\mu \eta}}{\Lambda^{2}}\right\} \overline{\mu_{R}} \sigma^{\alpha \beta}\left(H \ell_{\eta}\right) F_{\alpha \beta}+\left\{\frac{C^{\eta \mu}}{\Lambda^{2}}\right\} \overline{e_{R \eta}} \sigma^{\alpha \beta}\left(H \ell_{\mu}\right) F_{\alpha \beta}+\text { h.c. } \quad(\eta=e, \mu)
$$

where $\sigma^{\alpha \beta}=\frac{i}{2}\left[\gamma^{\alpha}, \gamma^{\beta}\right]$. After Spontaneous Symmetry Breaking, the operator coefficient is multiplied by the Higgs vev $v$. In the SM, and any "Minimal Flavour Violating" (MFV) [5] extensions, this vev is accompagnied by a yukawa coupling, so one anticipates a suppression factor of the muon mass:

$$
\stackrel{S S B}{\longrightarrow} \frac{C^{\mu \mu} v}{\Lambda^{2}} \overline{\mu_{R}} \sigma^{\alpha \beta} \mu_{L} F_{\alpha \beta}+\text { h.c } \stackrel{M F V}{\longrightarrow} \frac{C m_{\mu}}{\Lambda^{2}} \bar{\mu} \sigma^{\alpha \beta} \mu F_{\alpha \beta}
$$

The real (imaginary) part of the flavour diagonal coefficient $C^{\mu \mu}$ is the magnetic (electric) dipole moment of the muon, written as:

$$
\stackrel{Q E D}{\longrightarrow}\left(e \frac{a_{\mu}}{4 m_{\mu}}+i \frac{d_{\mu}}{2}\right) \bar{\mu} \sigma^{\alpha \beta} \mu F_{\alpha \beta} \quad a_{\mu}, d_{\mu} \text { real }
$$

5. $(g-2) \mu$

The observed precession of the muon spin, due to its magnetic moment $g_{\mu}$ in $\vec{B}, \vec{E}$ fields, was measured at BNL, by experiment E821, to be [6]

$$
a_{\mu}=\frac{g_{\mu}-2}{2}=(11659208.0 \pm(5.4)(3.3)) \times 10^{-10} .
$$

Theoretical calculations [7] of $a_{\mu}$ in the Standard Model differ from the experimental value by $\sim 3-4 \sigma$. See the contribution by L Roberts, and [7] and references therein, for possibilities and prospects of improving the theory calculation.

The diagrams which contribute can be classified as 


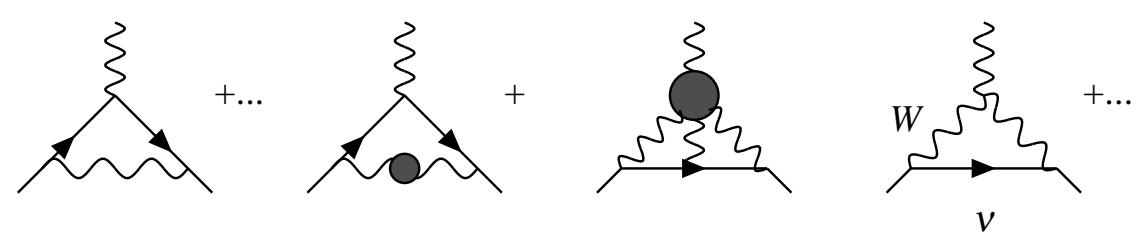

Figure 1: Representative diagrams contributing to $a_{\mu}$ in the SM.

- QED, which are the largest contribution, involving the electromagntic coupling. The one loop contribution is the diagram to the left in figure 1. The energy scale in the denominator is $m_{\mu}$, and the diagrams are known to better than 4 loops.

- QCD/hadronic, involving strongly interacting particles in the loop. These are the most problematic diagrams in the calculation. The vacuum polarisation contribution, which is the second diagram from the left in figure 1, can be obtained (with significant errors) with the optical theorem, which gives the imaginary part of the hadronic vaccum polarisation in terms of the total cross-section for $e^{+} e^{-} \rightarrow$ hadrons. Then the full matrix element may be obtained from its imaginary part by analyticity, which gives

$$
\left.\delta a_{\mu}\right|_{H P V}=\left(\frac{\alpha m_{\mu}}{3 \pi}\right)^{2} \int_{4 m_{\pi}^{2}}^{\infty} \frac{d s}{s^{2}} K(s) R(s)
$$

where $R=\sigma\left(e^{+} e^{-} \rightarrow\right.$ hadrons $) / \sigma\left(e^{+} e^{-} \rightarrow \mu^{+} \mu^{-}\right)$, and $\mathrm{K}(\mathrm{s})$ is a kinematic function.

The light-by light diagram, third in figure 1 must be estimated in models. See the contribution of L Roberts, or for instance [8].

- electroweak, of order $m_{\mu} / 16 \pi^{2} m_{W}^{2}$.

\subsection{Is this NP accessible to the LHC?}

In the case that the NP is Minimally Flavour Violating, and contributes to $g-2$ in a loop, one expects $a_{\mu}^{B S M} \sim \frac{C m_{\mu}^{2}}{4 \pi e M_{B S M}^{2}}$. This gives $M_{B S M}<\sqrt{C} \mathrm{TeV}$, so for $C<1$ (reasonable), NP with an electric charge could be produced at the LHC. This case includes many extensions of the SM that are consistent with flavour data, and contain a dark matter particle. A tree level NP contribution to $g-2$ is possible [9], although it may not seem motivated by other considerations.

If the NP is not MFV, the $h_{\mu}$ suppression of the dipole coefficient may be absent: $a_{\mu}^{B S M}=$ $\frac{C v m_{\mu}}{4 \pi e M_{B S M}^{2}}$, and the $g-2$ discrepancy can be fitted with $M_{B S M}<\sqrt{C} \times 30 \mathrm{TeV}$, which could be beyond the reach of the LHC. If such NP couplings were flavour universal, they would also contribute to the magnetic dipole moment of the electron, which determines $\alpha_{e m}$. However, the contribution would cause $\alpha_{e m}$ to deviate by only $\sim 1 \sigma$ from other determinations (cold atoms).

\subsection{Is it interesting to better measure $a_{\mu}^{t h}-a_{\mu}^{e x p t}$ ?}

A better determination of $a_{\mu}^{\text {expt }}$ is desirable in all scenarios, as discussed in [10]. The LHC may find the new particles responsable for $a_{\mu}^{\text {th }}-a_{\mu}^{\text {expt }}$. In this case, the best possible $g_{\mu}-2$ determination would contribute to the identification of the new physics. 
The $a_{\mu}^{\text {th }}-a_{\mu}^{\text {expt }}$ discrepancy could go away, following improvements in the measurement or the calculation. But the LHC could still find new physics. For instance, Little Higgs models with T-parity [16] give a negligeable contribution to $g_{\mu}-2$, but could be discovered at the LHC.

In the case that $a_{\mu}^{t h}-a_{\mu}^{e x p t}$ is due to new physics beyond the reach of LHC, it would be important to confirm the discrepancy, so as to better guess where to look for the NP. (If it is not MFV, it should show up in flavour physics? Or if it was flavour-universal, it should contribute also to $a_{e}$, which would make a better determination of $\alpha_{e m}$ interesting.)

6. $\mu \rightarrow e \gamma$

The dipole operator with leptons of different flavours, for instance eqn (4.1) with $\eta=e$, gives rise to $\mu \rightarrow e \gamma$ with $B R \simeq \frac{48 \pi^{2}}{\left|m_{\mu} G_{F}\right|^{2}}\left(\left|\frac{C^{\mu e_{v}}}{M_{B S M}^{2}}\right|^{2}+\left|\frac{C^{e \mu_{v}}}{M_{B S M}^{2}}\right|^{2}\right)<1.2 \times 10^{-11}$. The MEG experiment which is running now, should improve this sensitivity by a few orders of magnitude. With a different choice of flavour indices, this dipole operator mediates $\tau \rightarrow \mu \gamma\left(B R<6.8 \times 10^{-8}\right)$, and $\tau \rightarrow e \gamma$ $\left(B R<1.1 \times 10^{-7}\right)$, where the bounds on the branching ratios are from the $\mathrm{B}$ factories.

Although the leptonic mixing angles are large, the neutrino masses are very small, so the flavour changing contribution to the coefficient from neutrinos masses is GIM suppressed to irrelevance. Observable rates are therefore evidence for additional NP beyond $m_{v}$.

\subsection{Does $\mu \rightarrow e \gamma$ have any relation to $g-2$ ?}

Supppose that the $g-2$ discrepancy is due to NP: $a_{\mu}^{N P}=a_{\mu}^{t h}-a_{\mu}^{\text {expt }}$. To observe $\mu \rightarrow e \gamma$, it seems useful to have new particles with gauge interactions (also useful for $g-2$ ), and new flavour changing interactions. These two aspects can be separated by normalising $\mu \rightarrow e \gamma$ to $g-2$ [12]:

$$
\frac{C^{\mu e} v}{M_{B S M}^{2}}, \frac{C^{e \mu} v}{M_{B S M}^{2}}=\frac{C^{\mu \mu} v}{2 M_{B S M}^{2}} \theta_{\mu e}^{L, R} e^{i \delta_{L, R}}
$$

which gives $B R(\mu \rightarrow e \gamma) \simeq 10^{-3}\left[\delta a_{\mu}^{B S M} / 3 \times 10^{-9}\right]^{2}\left(\left|\theta_{\mu e}^{L}\right|^{2}+\left|\theta_{\mu e}^{R}\right|^{2}\right)$ where $L, R$ is the chirality of the outgoing electron. This normalisation is interesting in models which predict flavour-change, such as SUSY-GUTS (see e.g. [1]). It would give information on the flavour-changing parameters from observations of $\mu \rightarrow e \gamma$ and $g-2$.

\subsection{If $\mu \rightarrow e \gamma$ is seen - is more statistics interesting?}

The process is mediated by two operators with independent complex coefficients:

$$
\left\{\frac{C^{\mu e}}{\Lambda^{2}}\right\} \overline{\mu_{R}} \sigma^{\alpha \beta}\left(H \ell_{e}\right) F_{\alpha \beta}+\left\{\frac{C^{\mu}}{\Lambda^{2}}\right\} \overline{e_{R}} \sigma^{\alpha \beta}\left(H \ell_{\mu}\right) F_{\alpha \beta}+\text { h.c. }
$$

The coefficient $C^{\mu e}\left(C^{e \mu}\right)$ corresponds to an outgoing $e_{L}\left(e_{R}\right)$. In the "MFV" hypothesis, the $\mu$ Yukawa provides the chirality flip, so the NP is in the doublets (singlets).

Consider now the decay of a $\mu^{+}$, which is automatically polarised. Since chirality is helicity for relativistic electrons, the positron momentum will be preferentially aligned, or anti-aligned, with the muon spin, depending on which coefficient dominates the rate. An illustrative diagram can be found in [1]. So information on the relative magnitude of the two coefficients can be obtained from the angular distribution of the outgoing $e^{+}$. 


\subsection{What about the phases of the operator coefficients?}

In a local Lorentz-invariant theory, described by a unitary S-matrix (and respecting spin-stats), $C P T$ is a symmetry. However, this does not quite mean that $C P=T^{-1}$ when applied to states:

$$
C P:|\operatorname{particle}(\vec{p}, \vec{s})\rangle \rightarrow|\operatorname{antipart}(-\vec{p}, \vec{s})\rangle \quad T:|\operatorname{particle}(\vec{p}, \vec{s})\rangle \rightarrow\langle\operatorname{antipart}(-\vec{p},-\vec{s})|
$$

so for matrix elements

$$
\begin{array}{r}
C P: \mathscr{M}\left(\left\{\left(\vec{p}_{i}, \vec{s}_{i}\right)\right\} \rightarrow\left\{\left(\vec{p}_{f}, \vec{s}_{f}\right)\right\}\right) \longrightarrow \mathscr{M}\left(\left\{\left(-\vec{p}_{\bar{i}}, \vec{s}_{\bar{i}}\right)\right\} \rightarrow\left\{\left(-\vec{p}_{\bar{f}}, \vec{s}_{\bar{f}}\right)\right\}\right) \\
T: \mathscr{M}\left(\left\{\left(\vec{p}_{i}, \vec{s}_{i}\right)\right\} \rightarrow\left\{\left(\vec{p}_{f}, \vec{s}_{f}\right)\right\}\right) \longrightarrow \mathscr{M}\left(\left\{\left(-\vec{p}_{\bar{f}},-\vec{s}_{\bar{f}}\right)\right\} \rightarrow\left\{\left(-\vec{p}_{\bar{i}},-\vec{s}_{\bar{i}}\right)\right\}\right)
\end{array}
$$

If a triple product of $\vec{p}_{n}$ and $\vec{s}_{m}$ appears in the matrix element squared $|\mathscr{M}|^{2}$, (as can arise from $\operatorname{Tr}\left[\gamma_{0} \gamma_{i} \gamma_{j} \gamma_{k} \gamma_{5}\right]=4 i \varepsilon_{0 i j k}$ ), and if it is multiplied by complex coupling constants, there could be a $\mathrm{T}$ (or $\mathrm{CP}$ ) odd term in the differential rate. Information about the phases of coupling constants, could therefore be extracted from angular asymmetries in the differential rate. Notice that such asymmetries appears at tree level, and do not require measuring the difference between the rate for the process, and its time-reversed or CP conjugate.

As shown in [13], a non-zero triple product in $\mu \rightarrow e \gamma$ must involve both the photon and electron spins. If the $\gamma$ and $e^{+}$polarisations could be measured (eg $\vec{s}_{e}$ perpendicular to $\vec{p}_{e}$ ), then $\vec{s}_{\mu} \times \vec{s}_{e} \times \vec{s}_{\gamma} \neq 0$, and the "forward-backward" asymmetry

$$
A_{F B}=\Gamma(\mu \rightarrow e \gamma, \theta>\pi / 2)-\Gamma(\mu \rightarrow e \gamma, \theta<\pi / 2) \propto \operatorname{Im}\left[e^{i \phi_{s}} C^{\mu e} C^{e \mu *}\right] \sin \theta_{s}
$$

where $\vec{s}_{\mu} \cdot \vec{p}_{e} \propto \cos \theta, \vec{s}_{e} \times \vec{p}_{e} \propto \theta_{s}$, and $\vec{s}_{\gamma} \times \vec{p}_{\gamma} \propto \sin \phi_{s}$, could give the relative phase between the coefficients $C^{\mu e} C^{e \mu *}$.

\section{7. $\mu N \rightarrow e N$}

The decay $\mu \rightarrow e \gamma$ is searched for with $\mu^{+}$beams, which can be stopped in matter, where the muons decay. A $\mu^{-}$entering matter behaves differently. It gets bound to a nucleus, and cascades rapidly down to the $1 s$ state. From there, according to the SM, it can decay, or exchange a $W$ with the nucleus, which $\beta$ decays by muon capture: $\mu+(A, Z) \rightarrow v_{\mu}+(A, Z-1)$. In the presence of lepton flavour violating New Physics, the process $\mu+(A, Z) \rightarrow e+(A, Z)$ could also arise. It can be mediated by the dipole operators and by(many) 4-fermion operators:

$$
\bar{\mu} \sigma^{\alpha \beta}\left(\frac{C^{\mu e} v}{\Lambda^{2}} P_{L}+\frac{C^{e \mu} v}{\Lambda^{2}} P_{R}\right) e F_{\alpha \beta}+\sum_{\Gamma}\{(\bar{\mu} \Gamma e)(\bar{d} \Gamma d)+(\bar{\mu} \Gamma e)(\bar{u} \Gamma u)\}+\text { h.c. }
$$

Experimentally, the signature is a single $e^{-}$with $E \simeq m_{\mu}-E_{\text {bind }}$. This is less plagued by accidental backgrounds than the multi-body final state muon decays. The current bounds on Gold and Titanium [14], from SINDRUM II at PSI are :

$$
\frac{\Gamma(\mu A u \rightarrow e A u)}{\Gamma\left(\mu A u \rightarrow v A u^{\prime}\right)}<7 \times 10^{-13} \quad, \quad \frac{\Gamma(\mu A u \rightarrow e T i)}{\Gamma\left(\mu A u \rightarrow v T i^{\prime}\right)}<W H A T
$$

Next generation experiments with sensitivites $\rightarrow 10^{-18}$ are currently being planned at Fermilab $(\mu 2 e)$ and J-PARC (PRISM/PRIME), and are discussed in this volume. 


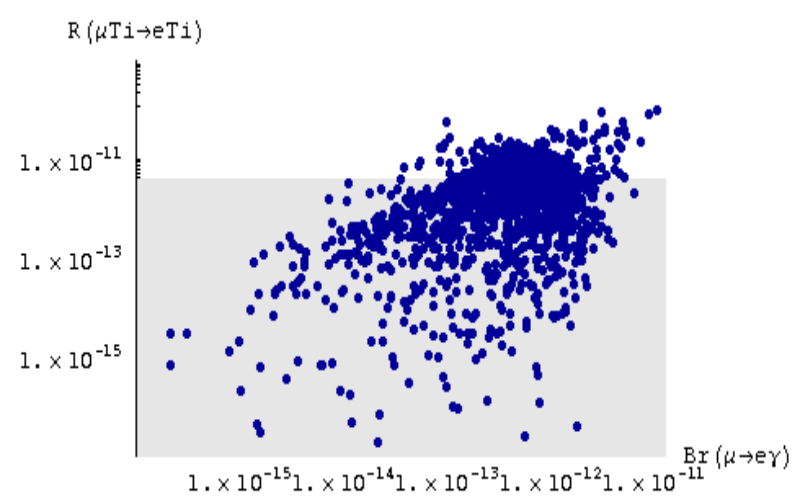

Figure 2: Figure taken from Blanke et al in [16], giving illustrative values of the branching ratios for $\mu \rightarrow e \gamma$ and $\mu-e$ conversion in the Little Higgs Model with T-parity.

\subsection{If $\mu \rightarrow e \gamma$ is observed, is $\mu N \rightarrow e N$ interesting?}

Observing $\mu \rightarrow e \gamma$ and $\mu-e$ conversion would allow to determine the relative importance of the 4-fermion and dipole operators. In certain NP scenarios, such as low $\tan \beta$ SUSY models, the $\mathrm{NP}$ contribution to the dipole coefficient are $\gg$ than to the 4-fermion coefficients. On then expects [17]

$$
\frac{B R(\mu \rightarrow e \gamma)}{B R(\mu N \rightarrow e N)}\left(\propto \frac{1}{\alpha}\right) \simeq \frac{428}{B(A, Z)} \quad B: 1.1 \rightarrow 1.8
$$

However, this relative suppression of the four fermion operators arises due to cancellations among NP box diagrams, which do not occur in many NP scenarios. For instance, in Little Higgs models with T-parity [16] there is no hierarchy between the 4-fermion and dipole coefficients, and the rates for $\mu \rightarrow e \gamma$ and $\mu-e$ conversion can be comparable (see fig 2).

\subsection{Is there more to learn from $\mu N \rightarrow e N$ than the rate?}

Various ideas have been put forward on how to disentangle the many possible operators contributing to $\mu-e$ conversion. They have different dependance on $A, Z$, so if the conversion rates are set equal on one nucleus, they can differ by a factor $\sim 1-2$ on a nucleus of different $Z$. See the discussion in [18], who showed that measuring the conversion rate on different nuclei could allow to distinguish operators.

If the muon was polarised, the angular distribution of the electron could give information on the "chirality" and phases of operator coefficients, as in $\mu \rightarrow e \gamma$ : for a polarised muon, $\vec{p}_{e} \cdot \vec{s}_{\mu}=$ \pm , distinguishes operators giving $e_{L}$ or $e_{R}$. However, the $\mu^{-}$is expected to retain $\sim 16 \%$ of its polarisation, after cascading down to the $1 s$ state (but it could be possible to restore $\mu^{-}$polarisation with a polarised target) [15].

\section{Summary: sharing the stage with the LHC}

If the LHC finds new physics, precision low energy observations will be crucial for obtain- 
ing model parameters to which LHC may not be sensitive (for instance flavour and CP violating couplings that could be measured in $B R(\mu \rightarrow e \gamma)$ ), and for model testing (perhaps $(g-2)_{\mu}$ will play the same role at the LHC, as $G_{F}$ did at LEP). And should the LHC have the misfortune to not find NP, most low energy experiments still could do so. One just must check what the LHC has excluded.

\section{References}

[1] Y. Kuno and Y. Okada, Rev. Mod. Phys. 73 (2001) 151 [arXiv:hep-ph/9909265].

[2] J. Aysto et al., arXiv:hep-ph/0109217.

[3] See, for instance, the reports at http://www.hep.ph.ic.ac.uk/iss/

[4] M. Raidal et al., arXiv:0801.1826 [hep-ph].

[5] G. D’Ambrosio, G. F. Giudice, G. Isidori and A. Strumia, Nucl. Phys. B 645 (2002) 155 [arXiv:hep-ph/0207036].

[6] G. W. Bennett et al. [Muon G-2 Collaboration], Phys. Rev. D 73 (2006) 072003 [arXiv:hep-ex/0602035].

[7] M. Passera, W. J. Marciano and A. Sirlin, arXiv:0809.4062 [hep-ph].

[8] J. Bijnens and J. Prades, Mod. Phys. Lett. A 22 (2007) 767 [arXiv:hep-ph/0702170].

[9] M. V. Chizhov, arXiv:0805.4313 [hep-ph].

[10] D. W. Hertzog, J. P. Miller, E. de Rafael, B. Lee Roberts and D. Stockinger, arXiv:0705.4617 [hep-ph].

[11] J. Hisano, AIP Conf. Proc. 981 (2008) 97.

[12] J. Hisano and K. Tobe, Phys. Lett. B 510 (2001) 197 [arXiv:hep-ph/0102315].

[13] Y. Farzan, JHEP 0707, 054 (2007) [arXiv:hep-ph/0701106].

[14] W. Bertl et al. [SINDRUM II Collaboration], Eur. Phys. J. C 47 (2006) 337.

[15] Y. Kuno, K. Nagamine and T. Yamazaki, Nucl. Phys. A 475 (1987) 615.

[16] S. R. Choudhury, A. S. Cornell, A. Deandrea, N. Gaur and A. Goyal, Phys. Rev. D 75 (2007) 055011 [arXiv:hep-ph/0612327]. M. Blanke, A. J. Buras, B. Duling, A. Poschenrieder and C. Tarantino, JHEP 0705 (2007) 013 [arXiv:hep-ph/0702136].

[17] A. Czarnecki, W. J. Marciano and K. Melnikov, AIP Conf. Proc. 549 (2002) 938.

[18] R. Kitano, M. Koike and Y. Okada, Phys. Rev. D 66 (2002) 096002 [Erratum-ibid. D 76 (2007) 059902] [arXiv:hep-ph/0203110]. R. Kitano, M. Koike, S. Komine and Y. Okada, Phys. Lett. B 575 (2003) 300 [arXiv:hep-ph/0308021]. 